\title{
EFECTIVIDAD DE UN PROGRAMA DE ESTIMULACION DE LA CONCIENCIA FONOLÓGICA EN NIÑOS DE 4 A 6 AÑOS
}

*Diana Margarita Van Leenden del Rio y Karen Lucia Lafaurie Lafaurie

** María Lucia S. Aparicio

\section{Resumen}

Esta investigación buscó establecer la efectividad de un programa que estimula la conciencia fonológica en niños de 4 a 6 años, que estudian en un colegio de educación regular. Fue aplicado por una profesora quien recibió previamente un taller de capacitación teórico-práctico por parte de las investigadoras para el desarrollo del mismo. El diseño utilizado fue preexperimental pre-prueba post-prueba con un solo grupo; la muestra fue de 14 niños, con edades comprendidas entre 4 años - 11 meses a 5 años - 10 meses, quienes cursan Transición en un colegio de la ciudad de Bogotá. Los niños fueron escogidos aleatoriamente, la evaluación inicial y final se realizó a través de la prueba de segmentación lingüística (PSL) que permite evaluar las distintas habilidades que tienen que ver con aislar, dividir, igualar y omitir unidades fonológicas del lenguaje. El periodo de aplicación fue de 2 meses. Los resultados arrojaron una diferencia estadísticamente significativa en todas las hipótesis, lo que permite constatar que el programa de estimulación de la conciencia fonológica es efectivo. Los datos se analizaron a través de la prueba " $t$ " de student para datos dependientes, con un alfa de 0.05.

Palabra Clave: Conciencia fonológica, Programa de Estimulación.

\section{INTRODICCIÓN}

Z1 desarrollo de la Conciencia Fonológica (CF), conocimiento de los segmentos fonoClógicos que componen la palabra, es uno de los aspectos a los que mayor atención se les ha prestado en los últimos años en el campo educativo.

\footnotetext{
" Estudiantes de Fonoaudiología, Escuela Colombiana de Rehabilitación

** Asesora Temática de la Investigación. Fonoaudióloga. Especialista en Docencia Universitaria.
} 
La relación entre la Conciencia Fonológica y el aprendizaje de la lecto-escritura es muy estrecha y hay evidencia que el conocimiento que tienen los niños sobre los componentes de la palabra y las estructuras gramaticales de las oraciones, facilitan el proceso de este aprendizaje.

La Conciencia Fonológica se refiere a la habilidad para reflexionar conscientemente sobre los segmentos fonológicos del lenguaje oral y a la toma de conciencia de cualquier unidad fonológica del lenguaje hablado; ya que este contiene algunas clases de unidades fonológicas, se hace necesario distinguir el conocimiento de tales unidades, y para esto se han considerado diferentes niveles de conciencia, teniendo en cuenta dos interpretaciones diferentes: la primera se refiere a que los niveles de Conciencia Fonológica se establecen de acuerdo con la dificultad de tareas; Leong (1991), distingue entre tareas de clasificación o emparejamiento y tareas de segmentación (aquellas que requieren la reproducción o manipulación de elementos aislados) atribuyendo una mayor facilidad para las tareas de clasificación.

Carrillo (1994) refiere dos componentes de Conciencia Fonológica: sensibilidad a las similaridades fonológicas (sensibilidad a la rima y al onset, y contar, aislar o detectar la posición de los fonemas), y conciencia segmental (omitir o invertir fonemas). Adams (1990) diferencia hasta 5 niveles de dificultad en las tareas que miden Conciencia Fonológica, estas van en orden de dificultad: recordar rimas familiares, reconocer y clasificar patrones de rima y aliteración en palabras, tareas de recomposición de sílabas en palabras, o de separación de algún componente de la sílaba (aislar el fonema inicial), segmentación de palabras en fonemas, añadir, omitir o invertir fonemas y producir la palabra o pseudo palabra resultante.

La segunda interpretación entiende que la Conciencia Fonológica no es una entidad homogénea porque se refiere a la conciencia de diferentes unidades lingüísticas, lo que permite que se hable de diferentes niveles de Conciencia Fonológica en función de unidad lingüística y objeto de recepción y manipulación por parte del niño. Según Jiménez (1995) diferencia tres niveles de conciencia: conciencia silábica, conciencia intrasílabica, conciencia fonémica. La conciencia silábica se refiere a la habilidad para segmentar, identificar o manipular, conscientemente las sílabas que componen una palabra. La conciencia intrasílabica se define como la habilidad para segmentar las sílabas en sus componentes intrasílabicos de onset y rima. El Onset es una parte integrante de la sílaba construída por la consonante o bloque de consonantes iniciales, los onsets de dos segmentos consisten en una única oclusiva o fricativa seguida por una de las líquidas $l$ o $r$. La rima es el constituyente obligatorio que tiene el pico de sonoridad y que tiene su propia estructura interna. El último y tercer nivel de conciencia, la fonémica, se refiere a la habilidad metalingüística e implica la comprensión de las palabras habladas estan constituídas por unidades sonoras discretas que son los fonemas. Es decir, la habilidad para prestar atención consiste en reconocer los sonidos como unidades abstractas y manipulables.

Algunos autores afirman que la Conciencia Fonológica se desarrolla entre $\operatorname{los} 4$ y 5 años; mientras que otros la sitúan entre los 6 y 7 años. Durante el desarrollo de la Conciencia Fonológica se puede observar que la conciencia silábica precede a la conciencia fonémica, las tareas de omisión de consonantes en posición inicial y media resulta ser más difícil en niños en edad preescolar, también se puede ver que contar sílabas es mucho más fácil que contar fonemas debido a que los fonemas aparecen coarticulados en las palabras. En conclusión, los niños primero desarrollan la conciencia de sílabas, luego el conocimiento de unidades intrasilábicas (onset-rima) y finalmente el conocimiento fonémico.

La Conciencia Fonológica está plenamente relacionada con la habilidad lectora y la habilidad para la escritura. Es importante tener en cuenta que la operación de la lectura se realiza sobre un sistema de escritura que representa la estructura sonora del lenguaje hablado, los sistemas de escritura alfabéticos exigen una ruta fonológica que luego va a permitir acceder 
al significado. En las primeras etapas del aprendizaje del código lecto-escrito es necesario ser capaz de segmentar y tomar conciencia de las unidades que constituyen el lenguaje oral.

Es evidente que el aprendizaje de la lectura y la escritura exige un esfuerzo metalingüístico, ya que, si nuestro sistema de escritura representa la estructura fonológica del habla, es necesario que los niños accedan primero al código fonológico y pueda resultar más fácil después poner en relación las unidades sonoras y gráficas (Alegría, 1985). Esto no significa que los niños tengan que ser plenamente concientes de la estructura fonológica del lenguaje antes de aprender el lenguaje escrito, por el contrario se sugiere que los niños adquieran un nivel mínimo de Conciencia Fonológica para que sea más fácil la adquisición de las correspondencias letra-sonido y descomponer el código ortográfico. Teniendo en cuenta lo mencionado acerca de la Conciencia Fonológica el problema planteado en este estudio fue: ¿Es posible estimular la Conciencia Fonológica en niños de 4 a 6 años a través de un programa de estimulación?.

\section{MÉtodo}

El diseño que se utilizó fue de tipo preexperimental pre-prueba post-prueba con un grupo de niños que recibieron un programa de estimulación de la Conciencia Fonológica.

Las hipótesis se plantearon teniendo en cuenta la Prueba de Segmentación Lingüística (PSL) realizada por Juan E. Jiménez González y María del Rosario Ortiz González; los ítemes que evalúa son:

- Descubrir palabras que no riman: consiste en la habilidad para descubrir qué pares de palabras no tienen una terminación fonética similar, ya que no coincide la sílaba final.

- Omisión de sílabas en posición inicial en palabras bisílabas: este hace referencia a la habilidad para omitir, en el momento de pronunciar el nombre de un dibujo que contiene dos sílabas, la primera sílaba CV.

- Segmentar palabras trisílabas: consiste en la habilidad para identificar el número de sílabas que contiene una serie de palabras trisílabas.

- Omisión de sílabas en posición inicial en las palabras trisílabas:consiste en la habilidad para omitir, en el momento de pronunciar el nombre del dibujo que contiene tres sílabas, la primera $\mathrm{CV}$.

- Descubrir palabras que coinciden en la sílaba final: se refiere a la habilidad de descubrir qué pares de palabras tienen una terminación fonética similar al coincidir la sílaba final.

- Omisión de sílabas especificadas previamente en posición final en palabras bisílabas: da cuenta de si el alumno es capaz de omitir, en el momento de pronunciar el nombre de un dibujo que contiene dos sílabas, la última sílaba VC.

- Recomponer palabras trisílabas: que consiste en la habilidad para descubrir qué palabra se forma al escuchar previamente las sílabas que la forman con un intervalo temporal entre cada una de ellas.

- Segmentar palabras bisílabas: Se refiere a la habilidad para identificar el número de sílabas que contienen una serie de palabras bisílabas.

- Omisión de sílabas en posición final en palabras bisílabas:consiste en la habilidad para omitir en el momento de pronunciar el nombre de un dibujo que contiene dos sílabas, la última sílaba es $\mathrm{CV}$.

- Segmentar oraciones con nexos: hace referencia a la habilidad del alumno para dividir oraciones que contienen palabras de clase abierta y cerrada.

- Omisión de sílabas en posición final en palabras trisílabas: consiste en la habilidad para omitir en el momento de pronunciar el nombre de un dibujo que contiene tres sílabas, la ultima sílaba CV.

- Aislar sílabas: se refiere a la habilidad para detectar aquellos dibujos cuyos nombres comienzan o terminan por un sonido vocálico o sílaba determinada.

- Descubrir palabras que coinciden en la sílaba inicial: consiste en la habilidad para descubrir qué pares de palabras tienen en común la sílaba inicial.

- Segmentar oraciones sin nexos: se refiere a la habilidad del alumno para dividir oraciones que sólo contienen palabras de clase abierta.

- Aislar sonidos consonánticos: se refiere a la habi- 


\section{ARTICULOS DE TESIS DE INVESTIGACIÓN}

lidad para detectar aquellos dibujos cuyos nombres comienzan o terminan por un sonido consonántico.

- Omisión de sílabas especificadas previamente en posición inicial en palabras trisílabas: este hace referencia a la habilidad para omitir, en el momento de pronunciar el nombre de un dibujo que contienen tres sílabas la sílaba CV.

Para el desarrollo de esta investigación se tomaron 14 niños, que se encuentran cursando Transición, con edades entre los 4 años y 11 meses a 5 años y 10 meses, compuesto por 9 niñas y 5 niños que recibieron el programa de estimulación de Conciencia Fonológica.

Tabla 1. Características de la población en edad y género.

\begin{tabular}{|c|c|c|c|}
\hline & EDAD & Masculino & Femenino \\
\hline Sujeto 1 & 5 años 1 mes & 1 & \\
\hline Sujeto 2 & 5 años 10 meses & & 1 \\
\hline Sujeto 3 & 5 años 8 mes & & 1 \\
\hline Sujeto 4 & 4 años 11 meses & & 1 \\
\hline Sujeto 5 & 5 años 9 meses & & 1 \\
\hline Sujeto 6 & 5 años 10 meses & 1 & \\
\hline Sujeto 7 & 5 años 8 meses & & 1 \\
\hline Sujeto 8 & 5 años 2 meses & 1 & \\
\hline Sujeto 9 & 5 años 3 meses & 1 & \\
\hline Sujeto 10 & 5 años 1 mes & & 1 \\
\hline Sujeto 11 & 5 años 6 meses & & 1 \\
\hline Sujeto 12 & 5 años 9 meses & & 1 \\
\hline Sujeto 13 & 5 años 6 meses & & 1 \\
\hline Sujeto 14 & 5 años 1 mes & & 1 \\
\hline
\end{tabular}

La profesora que llevó a cabo la aplicación no se encontraba informada de la evaluación inicial de los niños, de esta forma no se realizó ninguna modificación del criterio ni preferencia en la aplicación del programa para ningún niño.

Para la realización de esta investigación se contó con:

1. Taller informativo teórico práctico creado por las investigadoras, donde se encuentra información relevante sobre la conciencia fonológica.

2. Prueba de Segmentación Lingüística (PSL), realizada por Juan E. Jiménez González y María del Rosario Ortiz González. Se trata de una prueba de aplicación individual. Se realizan dos sesiones de 45 minutos de duración indicada especialmente para llevar a cabo una evaluación de la Conciencia Fonológica durante el período de educación infantil.

Esta prueba tiene un coeficiente de confiabilidad de 0.97 y su validez presenta correlaciones estadísticamente significativas con la comprensión lectora y con el número de errores en la tarea de decodificación grafémico-fonémico tanto en palabras como en pseudopalabras.

3. Programa de estimulación de la Conciencia Fonológica creado por las investigadoras.

\section{Procedimiento}

Para esta investigación se tuvieron en cuenta las siguientes fases:

- Fase 1: se contactó el colegio y la profesora quien realizó la aplicación del programa de estimulación de la Conciencia Fonológica.

- Fase 2: se seleccionaron 14 niños a los cuales se les aplicó el programa y la evaluación, teniendo en cuenta que las edades estuvieran entre los 4 y 6 años.

- Fase 3: se creó el programa de estimulación teniendo en cuenta las distintas habilidades que tienen que ver con aislar, dividir, igualar y omitir unidades fonológicas del lenguaje.

- Fase 4: se capacitó a la profesora de preescolar con el taller teórico práctico por un tiempo de 45 minutos y fue entregado el programa de estimulación.

- Fase 5: se realizó el pre-test a través de la prueba PLS, con las condiciones de aplicación establecidas por dicho instrumento.

- Fase 6: se llevó a cabo la aplicación del programa 
de estimulación de la Conciencia Fonológica por parte de la profesora, por un tiempo de 2 meses; las actividades tenían una duración de 30 minutos cada una.

- Fase 7: se realizó el post-test al grupo a través de la prueba PLS, con las condiciones de aplicación establecidas por la misma.

- Fase 8: se organizaron y compararon los datos con el fin de verificar las hipótesis y los objetivos, aspectos que serán analizados con los resultados de esta investigación.

\section{Resultados}

Para el análisis de los resultados se realizó un pretest y un post-test a cada uno de los participantes del estudio teniendo en cuenta los objetivos y cada una de las variables con sus respectivas hipótesis ya mencionadas anteriormente. Respecto al objetivo general de esta investigación se puede establecer que se cumplió, ya que se observó una diferencia significativa en el desarrollo de la habilidad de la Conciencia Fonológica después de la aplicación del programa de estimulación.

De acuerdo con los resultados arrojados en la prueba $\boldsymbol{t}$ de Student aplicada a cada hipótesis se encontraron cambios significativos a nivel estadístico, así como también se rechazaron todas las hipótesis nulas propuestas. La investigación reportó resultados favorables puesto que las hipótesis tomaron la dirección esperada. Esta evidencia anteriormente mencionada se pudo comprobar, ya que variables como la segmentación y omisión lograron desarrollarse de una manera efectiva en los niños al finalizar el programa de estimulación de la Conciencia Fonológica. Se observó también cómo en estos niños se logra una mayor concentración y desempeño cuando se realizan actividades en forma de juego en las cuales no se sienten bajo presión de obtener una calificación

\section{Discusión}

Esta investigación se realizó luego de detectar la necesidad de desarrollar en los niños la capacidad de conocer y analizar la estructura fonológica de la lengua, porque sin duda alguna, la ausencia de la Conciencia Fonológica, es un factor explicativo de las dificultades de aprendizaje que presentan los niños en el proceso de la lecto-escritura como lo corroboran las investigaciones de Fisher y Carter (1974) quienes condujeron que los niños preescolares que tenían dificultad en la Conciencia Fonológica en el futuro no se constituyen en buenos lectores.

La estimulación de la Conciencia Fonológica en niños de preescolar es un trabajo que arroja resultados positivos para el aprendizaje de la lecto-escritura. Lo anterior se evidenció después de realizar las actividades del programa de estimulación sugerido, determinando los cambios significativos en cada una de las hipótesis analizadas. Estas hipótesis se observaron a través de la Prueba de Segmentación Lingüística (PLS).

En la Conciencia Fonológica se trabajan distintas actividades, que tienen que ver con aislar, dividir, igualar y omitir unidades fonológicas del lenguaje. La evaluación de estas variables se concretó a través de la prueba objetiva, Prueba de Segmentación Lingüística (PSL); por medio de un pre-test y un post-test efectuado por las mismas evaluadoras en las dos sesiones, donde a cada uno de los participantes se les realizó una idéntica evaluación. Cada una de las evaluaciones, fue aplicada en un tiempo de 45 minutos, las sesiones se realizaron en dos momentos diferentes y de forma individual. En la primera sesión se administraron las tareas I, II y III, y, en la segunda, las tareas IV, V, VI y VII, las cuales contienen cada uno de los ítemes o variables que fueron analizados anteriormente.

Este instrumento está diseñado especialmente para llevar a cabo una evaluación de la Conciencia Fonológica durante el período de la educación infantil. Se trata de una prueba objetiva y estandarizada que incluye fundamentalmente tareas como segmentar palabras en sílabas, entre otras. Para este proceso se consideró importante la participación de la profesora de preescolar, teniendo en cuenta que ella está en contacto constante con los niños.

Este estudio plantea la necesidad de realizar una 
estimulación de la Conciencia Fonológica como prerequisito para adquirir adecuadamente el proceso de la lecto-escritura, de tal modo, que ayude a disminuir los problemas posteriores en estas áreas, es decir, un trabajo específico de la Conciencia Fonológica, teniendo en cuenta que ésta se encuentra plenamente relacionada con la habilidad lectora y la habilidad para la lecto-escritura.

Sobre la base de lo anterior se logró una efectividad del programa de estimulación ya que cada una de las actividades les brindó a los niños los medios necesarios para desarrollar la conciencia explícita de la estructura segmental de la palabra.

Es importante señalar que si bien es cierto, algunas de estas actividades programadas son trabajadas en ocasiones dentro del aula de clase, no se realizan con la misma frecuencia ni énfasis y no se les da la importancia necesaria.

Se comprobó que las variables como la segmentación y omisión lograron desarrollarse de una manera efectiva en los niños al finalizar el programa de estimulación de la Conciencia Fonológica. Se observó también cómo en estos niños se logra una mayor concentración y desempeño cuando realizan actividades en forma de juego en las cuales no sienten presión de obtener una calificación.

Entre los aspectos tenidos en cuenta para la aplicación del programa y que definitivamente ayudaron a los resultados, está el tiempo de aplicación, el cual fue de dos meses de manera diaria y continuada, a la misma hora.

Siendo más puntuales en los resultados de esta investigación, se enfatiza en que se rechazaron todas las hipótesis nulas, ya que se encontró un aumento significativo en las habilidades evaluadas luego del programa de estimulación de la Conciencia Fonológica.

Analizando los resultados globales se encontró que los niños lograron un aumento significativo en todas las hipótesis obteniendo un puntaje total en pre-test de 19.29 y en el post-Test de 68.21. También se encontró un aumento marcado en las hipótesis F1 y F2 la primera corresponde a descubrir palabras que no riman (F1); en pre-Test de 1.93 y en el post-Test de 10.9, y la segunda que corresponde a la omisión de sílabas en posición inicial en palabras bisílabas (F2), con un puntaje de 0.57 en el pre-Test y en el post-Tes. de 7.29 ,

Lo anterior corrobora lo que Braeley y Brayant (1985), afirmaron al decir que el entrenamiento en actividades de ritmo, segregación y aliteración durante el periodo de jardín infantil tiene un papel decisivo sobre el aprendizaje de la lecto-escritura en los dos primeros años escolares. Así pues, se trabajaron actividades que contenían los diferentes aspectos relacionados con rimas, segmentación silábica, omisión de sílabas y descubrimiento de palabras que coincidan con la sílaba inicial o final de otra palabra.

Cabe resaltar que la mayor ganancia de la aplicación del programa de estimulación y además de demostrar su efectividad, fue lograr concientizar a la profesora de preescolar acerca de la importancia de la realización del trabajo de la Conciencia Fonológica. Por tal razón es trascendental seguir apoyando al grupo educativo.

Este programa de estimulación de la Conciencia Fonológica, podría ser utilizado como protocolo de actividades en la etapa pre-escolar y de esta manera, el docente puede realizar una detección temprana de las posibles dificultades en las habilidades lecto-escritas de sus alumnos.

El problema que se tiene hoy en día, es que en pocas instituciones escolares se tiene la posibilidad de contar con un grupo de trabajo interdisciplinario y por lo tanto, con un Fonoaudiólogo que pueda realizar un diagnóstico en esta área en los niños de pre-escolar, por tal razón cabe resaltar la importancia de implementar este programa de estimulación de la conciencia fonológica como herramienta básica para el trabajo pedagógico en el aula.

Los resultados obtenidos en este estudio demuestran que, luego de estimular cada uno de los aspectos que componen la Conciencia Fonológica, éstos se desarrollan efectivamente. Es importante insistir en que sean trabajados en su totalidad, ya que aquí radica el éxito del programa. Este estudio representó también 
un aporte valioso a la fonoaudiología y al ámbito pedagógico, no sólo por los resultados positivos, sino por ser una estrategia que contribuye al aprendizaje y al desarrollo de la lecto-escritura y brinda una oportunidad de actualización profesional.

\section{Conclusiones $Y$ Recomendaciones}

La relación de la Conciencia Fonológica y el aprendizaje de la lecto-escritura es muy estrecha y hay evidencia que el conocimiento que tienen los niños sobre los componentes de las palabras y las estructuras gramaticales de las oraciones, facilita el proceso de este aprendizaje. Sin embargo, hoy en día es poco lo explorado en este campo.

La evidencia anteriormente mencionada, se pudo comprobar ya que variables como la segmentación y omisión lograron desarrollarse de una manera efectiva en los niños al finalizar el programa de estimulación de la Conciencia Fonológica. Se observó también cómo en estos niños, se logra una mayor concentración y desempeño cuando se les realizan actividades en forma de juego en las cuales no se sienten bajo presión de obtener una calificación.

Estudios como éstos arrojan un fundamental aporte que se refleja en la necesidad de insistir a los profesores de preescolar para que realicen un trabajo formal y obligatorio de la Conciencia Fonológica en su currículo. Además éste trabajo plantea diferentes actividades como nuevas estrategias para trabajar en el salón de clase, creando así mayores expectativas en un campo poco explorado, brindando nuevas posibilidades al manejo del problema del aprendizaje de la lecto-escritura y sirviendo de medio de interacción entre el profesor y el alumno.

Siendo este programa de carácter grupal, permitió también que se diera una participación individual, donde cada uno de los niños, aportaba al desarrollo de la actividad en equipo logrando así, que se fomentara un interés personal.

Esta participación fue activa en la mayoría de los niños gracias a diferentes aspectos como: la labor y entrega por parte la profesora de pre-escolar, quien demostró en cada momento una firme convicción de la efectividad del programa de estimulación de la Conciencia Fonológica; otro de los factores que contribuyó al resultado positivo del programa de estimulación de la Conciencia Fonológica, fue el uso de diferentes materiales allí como: silbato, tambores, pelota y láminas con cada una de las letras del alfabeto, que motivaron y facilitaron el desarrollo de las tareas propuestas.

Esta investigación permitió conocer las diferentes maneras de asumir este trabajo para el desarrollo de ésta habilidad por las distintas profesiones que intervienen en el manejo integral del niño pre-escolar. Lo anterior explica que aunque el profesor sí trabaja algunas actividades relacionadas con el desarrollo de la Conciencia Fonológica, no son concientes de la identidad de dicha área ni de todos los aspectos que la integran.

Este análisis lleva a concluir que es indispensable realizar primero una capacitación al cuerpo docente con el fin de integrar y unificar conceptos y conocimientos que permitan desarrollar adecuadamente la intervención propuesta.

Estudios como estos generan un aporte fundamental al trabajo en equipo, promoviendo el crecimiento en las diferentes ramas educativas, fundamentalmente dirigidas a mejorar la calidad del aprendizaje de los niños, especialmente en aquellos que se encuentran en alto grado de riesgo.

Por todo lo anterior, se sugiere continuar con el trabajo de promoción y prevención en éste tema y no esperar a que los niños presenten una dificultad de aprendizaje en su proceso lecto-escrito, para trabajar éste aspecto y así arriesgarnos a que el problema sea aún más difícil de superar.

Resta mucho trabajo para lograr un óptimo uso de este tipo de recursos por parte de los equipos interdisciplinarios que intervienen en la educación y es una responsabilidad de profesionales en fonoaudiología difundir los beneficios de la aplicación y estudios de nuevas perspectivas de intervención que favorezcan el aprendizaje escolar. 


\section{Referencias Bibliográficas}

Adams, M.(1990). Beginning to read: Thinking and learning about print. Cambridge. MA.MIT Press.

Alegría, J. (1985). Por un enfoque psicolinguistico del aprendizaje de la lectura y sus dificultades. Infancia y aprendizaje.

A.P.N.E.D (1987). Psicología, lenguaje y aprendizaje. Editorial Panamericana S.A. Buenos Aires Argentina.

Azcoaga Juan(1981). Aprendizaje fisiológico y aprendizaje pedagógico. Editorial el Ateneo. Buenos Aires Argentina.

Azcoaga J.E y Cols(1981). Retardos del lenguaje en los niños. Editorial Paidos. Buenos Aires Argentina.

Bravo Valdivieso Luis(1999). Lenguaje y Dislexia. Editorial Universidad Católica. Santiago de Chile.

Bakker, (1992). Neuropsychological classification and treatment of dislexia. Journal of Disabilities, 25: 102-109

Bryant. P y Bradley. L (1983).Auditory organization and backwardness in reading. En: Rutter M (Ed.) Developmental neuropsychiatry. Nueva York. The Guilford Press.

Cardona. M. (1998).La conciencia metalingüística en el aprendizaje de la lecto-escritura.

Carrillo.M (1994).Development of phonological awareness and reading acquisition. A study in Spanish language. Reading and writing: an interdisciplinary Journal.

Catts.H (1991). Early identification of dyslexics: Evidence from a follow-up study of speech-language impaired children. Annals of Dyslexia, 41: 163-177

Condemarín Mabel(1993) La Lectura: Teoría, Evolución y Desarrollo. Editorial Andrés Bello. Santiago de Chile.

Fisher W. y Carter (1974).Explicit syllable and phoneme segmentation in the young child. Journal of Experimental Child Psychology, 18: 201-212

Fletcher, Flinn en (1996). Visual-Perceptual and phonological factors in the acquisition of literacy among children with congenital developmental coordination disorder. Dev-MedChild-Neurol 1997 Mar:39 (3): 158-66

Gonzalez, Jimenez J en (1998). Conciencia Fonológica y aprendizaje de la lectura. Editorial síntesis.

Grigorenko (1975) Developmental Dyslexia Journal of Child Psychol Psychiatry

Jiménez.J y Haro.C.(1995) Effects of Word Linguistic Properties on Phonological Awareness in Spanish Children. Journal of Educational Psichology.

Kamhi, (2000). The role of the speech-language pathologist in improving decoding skills. Semin-speech-lang.

Leong, CH.K.(1991).From phonemic awareness to phonological processing to language access in children developing reading proficiency. In D.J Sawyer y B.J Fox. New York.

Liberman I. y Shankweiler D.(1971).Letter confusions and reversals of sequence in the beginning reader. Cortex, 7 : 127-142.

McBride.Chang(1985) Models of Speech Perception and Phonological Processing in Reading. Child Dev.

Nopola. Henni (1998), A dominat gene for developmental dislexia on chromosome 3. J-Med-Genet.2001 Oct.38:64658

Quirós J.C y Cols(1980) Fundamentos Neuropsicología de las
Discapacidades del Aprendizaje. Editorial Panamericana S.A. Buenos Aires Argentina.

Olofsson.(2000) Naming Speed, Phonological Awareness and the Initial Stage of Learning to Read. Logoped Phoniatr vocol

Share D. y Col (1984). Sources of individual differences in reading acquisition. Journal of Educational Psychology, 76: 1309-1324

Stanovich K.E.(1984). Explaining the variance in reading ability in terms of psychological processes: What have we learned? Annals of Dislexia, 35: 67-96

Vellutino. (1995), Components of Reading ability: Implications for assessment and remediaton. Annual Conference on the American Speech-Language-Hearing Ass. California.

Vlugt Badian (1994), Do dyslexic and other poor readers differ in reading-related cognitive skills?. Reading and writing, 6 : 45-64 